\title{
Superiority of the 8th edition of the TNM staging system for predicting overall survival in gastric cancer: Comparative analysis of the 7th and 8th editions in a monoinstitutional cohort
}

\author{
HAO WANG ${ }^{*}$, WEIHONG GUO*, YANFENG HU, TINGYU MOU, LIYING ZHAO, \\ HAO CHEN, TIAN LIN, TUANJIE LI, JIANG YU, HAO LIU and GUOXIN LI
}

Department of General Surgery, Nanfang Hospital, Southern Medical University, Guangzhou, Guangdong 510515, P.R. China

Received January 22, 2018; Accepted July 24, 2018

DOI: $10.3892 / \mathrm{mco} .2018 .1683$

\begin{abstract}
The present study was performed to evaluate the predictive capacity of the 8th edition vs. the 7th edition of the American Joint Committee on Cancer (AJCC) tumor-nodemetastasis (TNM) staging system for overall survival (OS) of patients with gastric cancer. Data of eligible patients with gastric cancer in our institution between June 2004 and June 2014 were retrospectively reviewed. A total of 1,506 patients were followed up to July 2016, among whom 1,484 patients with complete stage information were included in the TNM staging analysis. A total of 339 (22.8\%) patients presented stage migration, including 325 (21.9\%) migrating to a lower tier and $14(0.9 \%)$ to a higher tier. All patients with stage migration to a lower tier were in stage III, including 177 (54.5\%) patients migrating from stage IIIB to IIIA, and 148 (45.5\%) from stage IIIC to IIIB. Patients migrating from IIIB to IIIA yielded a median OS time and 5-year OS rate closer to those remaining in stage IIIA. Similarly, patients migrating from IIIC to IIIB yielded a median OS time and 5-year OS rate closer to those remaining in stage IIIB. The 7th edition of the staging system exhibited prognostic discrepancy in discriminating stage IIIA from IIIB on survival curves, which was improved in the 8th edition. The 8th edition had a better predictive capability of
\end{abstract}

Correspondence to: Dr Guoxin Li or Dr Hao Liu, Department of General Surgery, Nanfang Hospital, Southern Medical University, 1838 North Guangzhou Avenue, Guangzhou, Guangdong 510515, P.R. China

E-mail: gzliguoxin@163.com

E-mail: liuhaofbi@163.com

*Contributed equally

Abbreviations: AJCC, American Joint Committee on Cancer; TNM, tumor-node-metastasis; OS, overall survival; MLNC, metastatic lymph node count; CEA, carcinoembryonic antigen; CA, cancer antigen; HR, hazard ratio

Key words: gastric cancer, American Joint Committee on Cancer, tumor-node-metastasis classification, 8th edition, survival survival, as evidenced by a smaller value of -2log likelihood in the Cox proportional regression model (7th edition 4738.859 vs. 8th edition 4736.683). Therefore, the present study demonstrated that the 8th edition of the AJCC TNM staging system is superior to the 7th edition in predicting the OS of patients with gastric cancer.

\section{Introduction}

Gastric cancer is one of the major causes of cancer-related mortality worldwide (1). The prognosis of gastric cancer varies by ethnicity, geographical region and disease severity at diagnosis. The tumor-node-metastasis (TNM) staging system has been validated as an effective tool for predicting the prognosis of malignant tumors, including gastric cancer (2). The first TNM classification for gastric cancer was published in the 2nd edition of the TNM Classification of Malignant Tumors in 1974 (3). Although some parts of the TNM staging system of gastric cancer remain controversial, with constant modifications it has become generally accepted and used to guide the management of gastric cancer worldwide.

The latest (8th) edition of the TNM classification was published in 2016 and replaced the 7th edition with several adopted modifications. In the 7th edition, the N3 category [metastatic lymph node count (MLNC) $\geq 7$ ] had been subdivided into N3a (MLNC 7-15) and N3b (MLNC $\geq 16$ ), but failed to incorporate this distinction into the final TNM staging (4). In the 8th edition, the N3 category is subdivided into N3a and N3b in the final pathological staging. Specifically, through this new classification, the T1N3bM0 of stage IIB was moved to IIIB, the T2N3bM0 of stage IIIA was moved to IIIB, the T3N3bM0 of stage IIIB was moved to IIIC, and the T4aN3aM0 of stage IIIC was moved to IIIB. Other modifications include $\mathrm{T} 4$ stage migrations, specifically $\mathrm{T} 4 \mathrm{bN} 0 \mathrm{M} 0$ and $\mathrm{T} 4 \mathrm{aN} 2 \mathrm{M} 0$ of stage IIIB moving to IIIA, and T4bN2M0 of IIIC moving to IIIB.

However, the necessity of these modifications remains unclear. In the present study, efforts were made to assess the prognostic significance of the 8th edition of the AJCC TNM staging system for gastric cancer. The aims were to: i) Evaluate the prognostic discrepancies in the presence or absence of the N3 category subdivision; ii) investigate the 
suitability of stage migrations, namely two subcategories (T4bN0M0 and T4aN2M0) moving from stage IIIB to IIIA and another subcategory (T4bN2M0) from stage IIIC to IIIB; and iii) compare the predictive ability of the 7 th vs. the 8 th staging system regarding overall survival (OS).

\section{Patients and methods}

Patients. The medical records of 1,525 patients with biopsy-confirmed gastric cancer at the Department of General Surgery of Nanfang Hospital (Guangzhou, China) between June 2004 and June 2014 were retrospectively reviewed from a prospectively collected database (5). Of the 1,525 patients, 19 were excluded, including 10 patients with carcinoma in situ, 5 patients with synchronous malignancies (1 with prostate cancer, 1 with Hodgkin's lymphoma, 1 with colon cancer, 1 with bladder cancer and 1 with nasopharyngeal cancer), 3 patients who died of postoperative complications, and 1 patient aged $<18$ years. Finally, 1,506 eligible patients were included in the subsequent analysis. The study was approved by the Ethics Committee of Nanfang Hospital of Southern Medical University. Written informed consent was obtained from all patients prior to entering their information into the database.

Surgical resection with a curative intent was performed for stage I-III patients and attempted for those with peritoneal metastasis, if allowed by preoperative assessment and intraoperative observation. Personalized adjuvant chemotherapy regimens were prescribed for patients, mainly using capecitabine, oxaliplatin, leucovorin and irinotecan. For patients with T3-T4 lesions or lymph node metastases, adjuvant chemotherapy was generally recommended. In this analyzed cohort, 656 (62.1\%) received adjuvant chemotherapy, whereas no patients received radiotherapy or chemotherapy as neoadjuvant treatment.

Methods. The patient demographic and clinicopathological data were analyzed, including gender (male or female), age ( $<60$ or $\geq 60$ years), history of abdominal surgery (yes or no), classification of comorbidities $(0,1$ or $\geq 2)$, Eastern Cooperative Oncology Group performance status $(0,1$ or $\geq 2)$, primary tumor size $(\leq 5$ or $>5 \mathrm{~cm})$, level of serum carcinoembryonic antigen (CEA) and cancer antigen (CA) 19-9 (normal or elevated), type of gastrectomy (subtotal or total), resection extent (radical or palliative), differentiation degree (high/ moderate or poor/signet-ring cell carcinoma) and positive resection margin (yes or no). Tumor classification was determined according to the 7th and 8th editions of the AJCC TNM staging system (referred to as 7th TNM and 8th TNM, respectively). For those patients with distant metastasis who were not considered eligible for surgical treatment, the pathological stage and $\mathrm{T}$ and $\mathrm{N}$ status were unavailable.

Statistical analysis. The aim of the present study was to evaluate whether the 8th TNM classification was superior to the 7th TNM classification in the prognostic prediction of patient OS. The OS time was defined as the duration from the date of surgery (or date of first diagnosis for those patients who did not undergo surgery) to the date of death or the last follow-up visit. The 5-year OS rate and median OS time were calculated, and survival curves were estimated using the Kaplan-Meier method. As mentioned above, the main modification in the 8th TNM staging system was the incorporation of $\mathrm{N} 3$ subdivisions (N3a and N3b) into the final pathological staging, which contributed to the shift of the final TNM classification. The discriminatory ability and monotonicity of gradient assessments were measured with the linear trend $\chi^{2}$ test of survival curves. Among the four aforementioned groups with migration, three groups (T3N3bM0, T2N3bM0 and T1N3bM0) included only a small number of patients, and the survival outcomes could not be statistically assessed. Since another group (T4aN3aM0) was a subgroup of the T4 stage, it was integrated with the other three T4 subgroups (T4bNOM0, $\mathrm{T} 4 \mathrm{aN} 2 \mathrm{M} 0$ and T4bN2M0) for analysis. The presence of the stage migration phenomenon mainly occurred in stage III, namely T4bNOM0 and T4aN2M0 of stage IIIB in the 7th TNM migrating to stage IIIA in the 8th TNM, and T4aN3aM0 and T4bN2M0 of stage IIIC in the 7th TNM migrating to stage IIIB in the 8th TNM. To investigate the suitability of these stage migrations, survival comparison was performed between patients with stage migration and those remaining in the same stage category according to both editions. The log-rank test and hazard ratio (HR) were used during analysis of univariate risk factors. Variables associated with OS were included in a multivariable Cox proportional regression model to identify independent risk factors of OS in the two staging systems. Variables highly related to others were excluded from the final model. Finally, two Cox regression models were built based on the 7th and 8th TNM to compare their prognostic prediction abilities. The variables in the two models were identical, except for TNM classification; in other words, the model based on the 8th TNM was constructed by replacing the 7th TNM stage by the 8th TNM stage. The statistics, -2log likelihood of the Cox regression model, were calculated to compare the predictive efficacy of the two models. A smaller value indicated a better model for predicting outcome.

For the majority of the patients, the postoperative follow-up was assessed at 3-month intervals for the first 2 years, 6-month intervals for the next 3 years, and annually thereafter until the endpoints were reached. Physical examination, serum tumor biomarker levels, chest X-ray, abdominal ultrasonography, gastroendoscopy and positron emission tomography/computed tomography were selected for accurate assessment at each follow-up. The last follow-up was performed in July 2016.

All statistical analyses were performed using SPSS version 17.0 for Windows (SPSS Inc., Chicago, IL, USA). A P-value of $<0.05$ (two-tailed) was considered to indicate statistically significant differences.

\section{Results}

Demographic and clinicopathological characteristics of the patients. Of the 1,506 patients included, 1,018 (67.6\%) were male and $488(32.4 \%)$ were female. The mean age of the entire cohort was 55.6 years (range, 19-90 years). The cohort was followed up for a mean duration of 44.1 months (range, 1-145 months). The demographic characteristics, pathological characteristics and clinical outcomes of the patients are summarized in Table I. The total number of dissected lymph nodes was 32,439 , with a mean \pm standard deviation of 
Table I. Demographics and univariate survival analysis results of the studied gastric cancer patients.

\begin{tabular}{|c|c|c|c|c|c|}
\hline \multirow[b]{2}{*}{ Variables } & \multirow[b]{2}{*}{ No. $(\%)$} & \multicolumn{3}{|c|}{$(95 \% \mathrm{CI})$} & \multirow[b]{2}{*}{$\begin{array}{c}\text { Log-rank } \\
\text { P-value }\end{array}$} \\
\hline & & 5 -year OS rate, $\%$ & $\begin{array}{l}\text { Median OS time, } \\
\text { months }\end{array}$ & Hazard ratio & \\
\hline Sex & 1,506 & & & & 0.434 \\
\hline Male & $1,018(67.6)$ & $47.4(44.1-50.7)$ & $55.0(43.7-66.3)$ & $0.943(0.812-1.094)$ & \\
\hline Female & $488(32.4)$ & $44.7(39.8-49.6)$ & $48.0(37.3-58.7)$ & (Reference) & \\
\hline Age (years) & 1,506 & & & & $<0.001$ \\
\hline$<60$ & $908(60.3)$ & $49.9(46.4-53.4)$ & $60.0(40.9-79.1)$ & (Reference) & \\
\hline$\geq 60$ & $598(39.7)$ & $41.3(36.8-45.8)$ & $38.0(29.1-46.9)$ & $1.306(1.133-1.505)$ & \\
\hline Abdominal surgery history & 1,506 & & & & 0.596 \\
\hline Yes & $153(10.2)$ & $45.3(36.5-54.1)$ & $49.0(20.9-77.1)$ & $1.064(0.844-1.341)$ & \\
\hline No & $1,353(89.8)$ & $46.7(43.8-49.6)$ & $54.0(46.3-61.7)$ & (Reference) & \\
\hline Classification of comorbidities & 1,506 & & & & 0.003 \\
\hline 0 & $1,149(76.3)$ & $47.2(44.1-50.3)$ & $54.0(44.2-63.8)$ & (Reference) & \\
\hline 1 & 269 (17.9) & $48.3(41.2-55.4)$ & $56.0(37.9-74.1)$ & $0.957(0.791-1.159)$ & \\
\hline$\geq 2$ & $88(5.8)$ & $28.8(16.6-41.0)$ & $30.0(15.2-44.8)$ & $1.559(1.188-2.407)$ & \\
\hline ECOG PS & 1,056 & & & & 0.461 \\
\hline 0 & 985 (65.4) & $82.6(0.018)$ & $139.0(128.1-149.9)$ & (Reference) & \\
\hline 1 & $350(23.2)$ & $79.7(0.038)$ & $84.4(68.2-100.7)$ & $1.274(0.978-1.506)$ & \\
\hline$\geq 2$ & $171(11.4)$ & $64.3(0.111)$ & $75.9(72.7-78.9)$ & $1.296(0.934-1.625)$ & \\
\hline Primary tumor size $(\mathrm{cm})$ & 1,344 & & & & $<0.001$ \\
\hline$\leq 5$ & $955(71.1)$ & $57.3(53.8-60.8)$ & $104.0(98.5-109.5)$ & (Reference) & \\
\hline$>5$ & $389(28.9)$ & $33.6(28.3-38.9)$ & $30.0(25.6-34.4)$ & $1.925(1.642-2.257)$ & \\
\hline Serum CEA & 989 & & & & $<0.001$ \\
\hline Normal & $832(84.1)$ & $48.1(44.6-51.6)$ & $56.0(44.3-67.7)$ & (Reference) & \\
\hline Elevated & 157 (15.9) & $29.0(21.4-36.6)$ & $22.0(15.2-28.8)$ & $1.726(1.400-2.127)$ & \\
\hline Serum CA 19-9 & 960 & & & & $<0.001$ \\
\hline Normal & $769(80.1)$ & $49.2(45.5-52.9)$ & $57.0(42.4-71.6)$ & (Reference) & \\
\hline Elevated & $191(19.9)$ & $28.5(21.6-35.4)$ & $20.0(16.3-23.7)$ & $1.903(1.566-2.313)$ & \\
\hline Gastrectomy type & 1,363 & & & & $<0.001$ \\
\hline Subtotal & $968(71.0)$ & $57.5(54.2-60.8)$ & $99.0(94.8-103.2)$ & (Reference) & \\
\hline Total & $395(29.0)$ & $34.0(28.5-39.5)$ & $32.0(26.8-37.2)$ & $1.932(1.644-2.271)$ & \\
\hline Resection extent & 1,363 & & & & $<0.001$ \\
\hline Radical & $1075(78.9)$ & $61.1(57.8-64.4)$ & Not reached & (Reference) & \\
\hline Palliative & $288(21.1)$ & $13.9(9.6-18.2)$ & $14.0(12.2-15.8)$ & $4.531(3.849-5.332)$ & \\
\hline Differentiation & 1,375 & & & & $<0.001$ \\
\hline High/moderate & $281(18.7)$ & $61.7(55.4-68.0)$ & Not reached & (Reference) & \\
\hline Poor/signet-ring cell & $1,094(79.6)$ & $44.2(40.9-47.5)$ & $48.0(40.7-55.3)$ & $1.746(1.413-2.158)$ & \\
\hline Positive resection margin & 1,363 & & & & $<0.001$ \\
\hline No & $1321(96.9)$ & $52.3(49.4-55.2)$ & $68.0(53.4-82.6)$ & (Reference) & \\
\hline Yes & $42(3.1)$ & $8.9(0.0-19.1)$ & $12.0(8.9-15.1)$ & $3.708(2.632-5.223)$ & \\
\hline Pathological T stage & 1,500 & & & & $<0.001$ \\
\hline $\mathrm{T} 1$ & $200(13.3)$ & $92.6(88.7-96.5)$ & Not reached & (Reference) & \\
\hline $\mathrm{T} 2$ & $118(7.9)$ & $74.7(66.3-83.1)$ & Not reached & $2.616(1.485-4.606)$ & \\
\hline $\mathrm{T} 3$ & $73(4.9)$ & $60.9(48.6-73.2)$ & Not reached & $5.268(2.938-9.446)$ & \\
\hline $\mathrm{T} 4 \mathrm{a}$ & $833(55.5)$ & $43.7(40.0-47.4)$ & $48.0(40.7-55.3)$ & $7.235(4.623-11.323)$ & \\
\hline $\mathrm{T} 4 \mathrm{~b}$ & $276(18.4)$ & $9.2(5.1-13.3)$ & $13.0(10.8-15.2)$ & $22.236(14.060-35.168)$ & \\
\hline Pathological N stage & 1,363 & & & & $<0.001$ \\
\hline N0 & $473(34.7)$ & $77.5(73.4-81.6)$ & Not reached & (Reference) & \\
\hline N1 & $237(17.4)$ & 55.7 (48.6-62.8) & Not reached & $2.196(1.665-2.896)$ & \\
\hline N2 & $287(21.1)$ & $42.2(36.1-48.3)$ & $47.0(33.2-60.8)$ & $3.340(2.612-4.271)$ & \\
\hline N3a & 244 (17.9) & $24.3(18.4-30.2)$ & $24.0(19.4-28.6)$ & $5.526(4.336-7.044)$ & \\
\hline $\mathrm{N} 3 \mathrm{~b}$ & $122(9.0)$ & $17.4(9.0-25.8)$ & $18.0(13.9-22.1)$ & $7.133(5.381-9.455)$ & \\
\hline
\end{tabular}


Table I. Continued.

$(95 \% \mathrm{CI})$

\begin{tabular}{|c|c|c|c|c|c|}
\hline \multirow[b]{2}{*}{ Variables } & \multirow[b]{2}{*}{ No. (\%) } & & \multirow[b]{2}{*}{$\begin{array}{c}\text { Log-rank } \\
\text { P-value }\end{array}$} \\
\hline & & 5 -year OS rate, $\%$ & $\begin{array}{l}\text { Median OS time, } \\
\text { months }\end{array}$ & Hazard ratio & \\
\hline 7th TNM stage & 1,484 & & & & $<0.001$ \\
\hline IA & $165(11.1)$ & $94.6(90.5-98.7)$ & Not reached & (Reference) & \\
\hline IB & $74(5.0)$ & $89.7(82.4-97.0)$ & Not reached & $1.430(0.584-3.498)$ & \\
\hline IIA & $62(4.2)$ & $81.9(71.3-92.5)$ & Not reached & $3.032(1.362-6.751)$ & \\
\hline IIB & $226(15.2)$ & $69.5(62.8-76.2)$ & Not reached & $4.331(2.345-8.002)$ & \\
\hline IIIA & $161(10.8)$ & $51.4(42.6-60.2)$ & $66.0(47.2-84.8)$ & $7.265(3.940-13.396)$ & \\
\hline IIIB & $202(13.6)$ & $47.3(39.7-54.9)$ & $57.0(38.3-75.2)$ & $8.561(4.708-15.567)$ & \\
\hline IIIC & $252(17.0)$ & $26.0(19.9-32.1)$ & $27.0(21.1-32.9)$ & $16.156(9.004-28.989)$ & \\
\hline IV & $342(23.0)$ & $9.4(5.9-12.9)$ & $11.0(9.6-12.4)$ & $35.797(20.060-63.879)$ & \\
\hline 8th TNM stage & 1,484 & & & & $<0.001$ \\
\hline IA & $165(11.1)$ & $94.6(90.5-98.7)$ & Not reached & (Reference) & \\
\hline IB & $74(5.0)$ & $89.7(82.4-97.0)$ & Not reached & $1.428(0.584-3.495)$ & \\
\hline IIA & $62(4.2)$ & $81.9(71.3-92.5)$ & Not reached & $3.037(1.364-6.762)$ & \\
\hline IIB & $224(15.1)$ & $69.7(63.0-76.4)$ & Not reached & $4.289(2.320-7.929)$ & \\
\hline IIIA & $334(22.5)$ & $49.8(43.7-55.9)$ & $60.0(38.8-81.2)$ & $7.716(4.290-13.880)$ & \\
\hline IIIB & $171(11.5)$ & $32.5(25.1-39.9)$ & $35.0(27.5-42.5)$ & 13.768 (7.598-24.949) & \\
\hline IIIC & $112(7.5)$ & $18.4(9.4-27.4)$ & $22.0(16.9-27.1)$ & $19.477(10.625-35.702)$ & \\
\hline IV & $342(23.0)$ & $9.4(5.9-12.9)$ & $11.0(9.6-12.4)$ & $35.994(20.169-64.233)$ & \\
\hline
\end{tabular}

OS, overall survival; CI, confidence interval; CEA, carcinoembryonic antigen; ECOG PS, Eastern Cooperative Oncology Group Performance Status; CA, cancer antigen.

23.8 \pm 19.3 . The mean number of metastatic lymph nodes was $5.2 \pm 7.7$ (median, 2; range, 0-65) in all patients and $8.0 \pm 8.3$ (median, 5; range, 1-65) in patients with lymph node involvement.

Survival comparison for $N$ category and stage migration. The main modification in the new TNM staging system was subdivision of the N3 category into N3a and N3b, and the resultant stage migration in the final TNM classification. The necessity of these modifications was unknown. In the present study, the OS curves based on the 7th TNM and 8th TNM were separately constructed and compared (Fig. 1). A log-rank test for trend revealed good discriminatory abilities in both $\mathrm{N}$ classifications (both $\mathrm{P}<0.001$ ), but the 8th TNM classification exhibited a better monotonicity of gradient compared with the 7th TNM classification, as evidenced by a higher $\chi^{2}$ value in the former classification ( $\chi^{2}$ for log-rank trend test, 311.783 vs. 297.588).

A total of 1,484 patients were included for analysis of TNM staging, and 22 patients with distant metastasis who did not undergo surgical resection were excluded due to unavailable data on $\mathrm{T}$ and $\mathrm{N}$ status. The distribution of patients according to the 7th and 8th editions of the AJCC TNM staging system is summarized in Table II. Among 1,484 patients, $1,145(77.2 \%)$ remained in the same stage category and 339 (22.8\%) migrated to a different stage, including $325(21.9 \%)$ patients who migrated to a lower tier and $14(0.9 \%)$ who migrated to a higher tier, compared with the 7th TNM. Given the small number of patients that migrated to a higher tier, including 2 patients in T1N3bM0 migrating from stage IIB to IIIB, 4 patients in $\mathrm{T} 2 \mathrm{~N} 3 \mathrm{bM} 0$ migrating from stage IIIA to IIIB, and 8 patients in $\mathrm{T} 3 \mathrm{~N} 3 \mathrm{bM} 0$ migrating from stage IIIB to IIIC, survival comparisons could not be made for these patients. Interestingly, all patients with stage migration to a lower tier were in stage III, including 177 (54.5\%) patients migrating from stage IIIB to IIIA, and $148(45.5 \%)$ patients migrating from stage IIIC to IIIB. To evaluate the suitability of these migrations, the OS of patients with stage migration was further compared to those remaining in the same stage category (Table III). For patients migrating from stage IIIB to IIIA, the survival curves had no significant difference from those that remained in stage IIIB or IIIA $(\mathrm{P}=0.342)$. However, patients with stage migration from IIIB to IIIA yielded a median OS time and 5-year OS rate closer to those remaining in stage IIIA (discrepancy of 8 months in median OS and 4.5\% in 5-year OS rate), compared with those remaining in stage IIIB (discrepancy of 21 months in median OS and $14.1 \%$ in 5-year OS rate). Similarly, for patients who migrated from stage IIIC to IIIB, the median OS and 5-year OS rate were closer to those remaining in stage IIIB, with a discrepancy of 4 months in median OS and $2.3 \%$ in 5-year OS rate, exhibiting a significant difference from those in stage IIIC (discrepancy of 13 months in median OS and $14.3 \%$ in 5-year OS rate; overall $\mathrm{P}=0.014)$. The discriminatory ability 
Table II. Distribution of patients according to the 7th and 8th editions of the AJCC staging system.

AJCC 8th stage

\begin{tabular}{lccccccccc}
\cline { 2 - 6 } AJCC 7th stage & IA & IB & IIA & IIB & IIIA & IIIB & IIIC & IV & Total \\
\hline IA & 165 & 0 & 0 & 0 & 0 & 0 & 0 & 0 & 165 \\
IB & 0 & 74 & 0 & 0 & 0 & 0 & 0 & 0 & 74 \\
IIA & 0 & 0 & 62 & 0 & 0 & 0 & 0 & 0 & 62 \\
IIB & 0 & 0 & 0 & 224 & 0 & $2^{\text {a }}$ & 0 & 0 & 226 \\
IIIA & 0 & 0 & 0 & 0 & 157 & $4^{\text {a }}$ & 0 & 0 & 161 \\
IIIB & 0 & 0 & 0 & 0 & $177^{\mathrm{a}}$ & 17 & $8^{\mathrm{a}}$ & 0 & 202 \\
IIIC & 0 & 0 & 0 & 0 & 0 & $148^{\mathrm{a}}$ & 104 & 0 & 252 \\
IV & 0 & 0 & 0 & 0 & 0 & 0 & 0 & 342 & 342 \\
Total & 165 & 74 & 62 & 224 & 334 & 171 & 112 & 342 & 1,484 \\
\hline
\end{tabular}

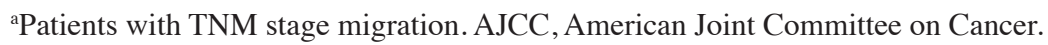

A

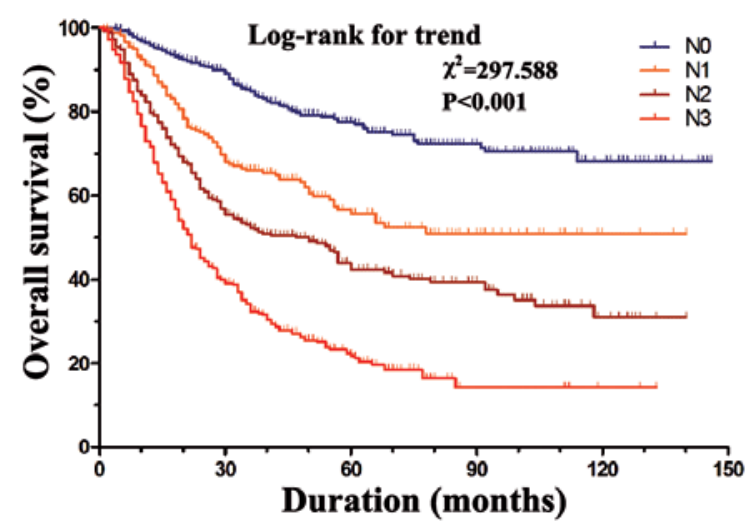

B

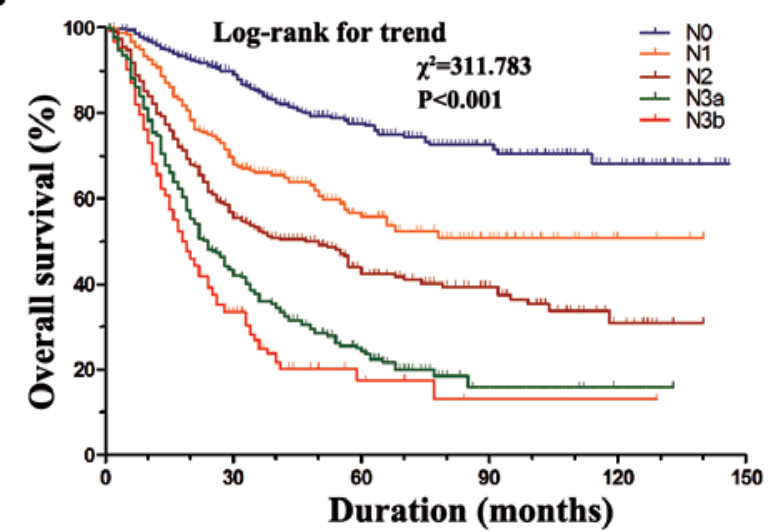

Figure 1. Kaplan-Meier survival curves for patients stratified by N category with (A) undivided N3 and (B) subdivided N3 into N3a and N3b. The log-rank test for trend showed significantly decreased survival in patients with advanced $\mathrm{N}$ stage by both $\mathrm{N}$ classifications (both $\mathrm{P}<0.001$ ), but the 8 th $\mathrm{TNM}$ classification exhibited a better monotonicity of gradient compared with the 7th TNM classification ( $\chi^{2}$ for $\log$-rank trend test, 311.783 vs. 297.588 ).
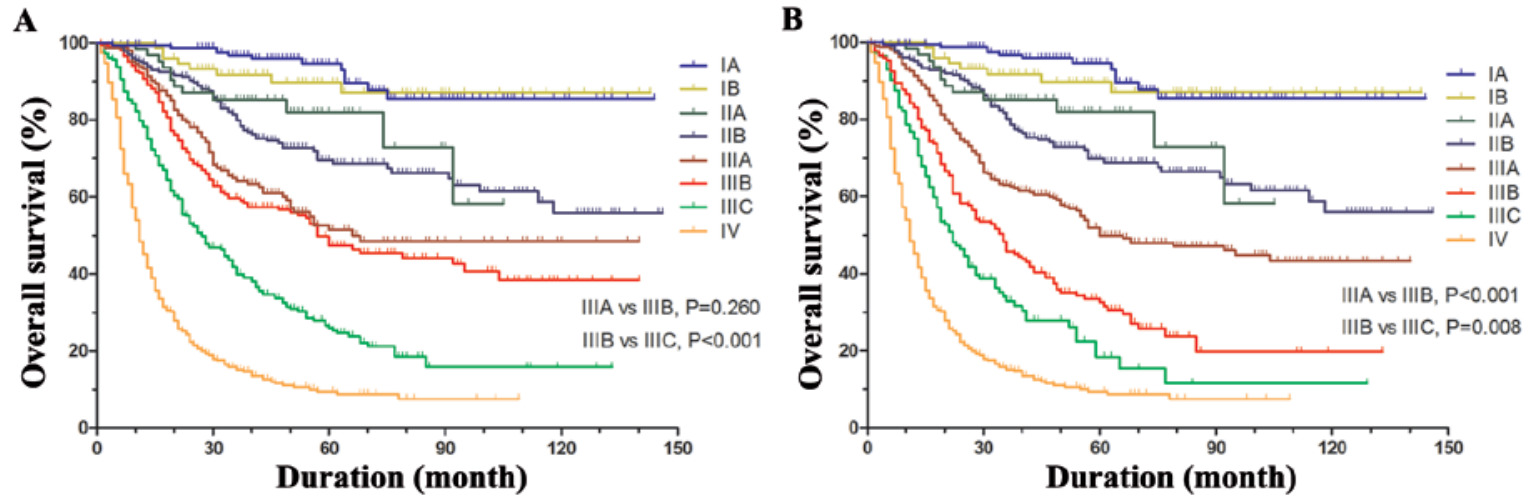

Figure 2. Kaplan-Meier survival curves for patients based on (A) the 7th AJCC staging system and (B) the 8th AJCC staging system. The 7th edition staging system exhibited insufficient prognostic discrepancy for discriminating stage IIIA from IIIB on survival curves ( $\mathrm{P}=0.260)$, which was improved in the 8 th edition $(\mathrm{P}<0.001)$. AJCC, American Joint Committee on Cancer.

in stage III was improved following stage migrations. The survival curves for stage IIIA and IIIB based on the 7th TNM were close $(P=0.260)$, with improved stratification in the 8 th edition $(\mathrm{P}<0.001)$. The survival curves of patients based on the 7 th and 8th editions of the AJCC TNM staging system are shown in Fig. 2. 
Table III. Survival comparison in stage III patients with stage migration.

\begin{tabular}{lcccc}
\hline Groups & Stage & Median OS (months) & 5-year OS rate (\%) & $\begin{array}{c}\text { Log-rank } \\
\text { P-value }\end{array}$ \\
\hline Migration from IIIB to IIIA $^{\mathrm{a}}$ & & & \\
Remaining in IIIA & T2N3aM0/T3N2M0/T4aN1M0 & $68.0(40.0-96.0)$ & $52.3(43.5-61.1)$ \\
IIIB to IIIA & T4bN0M0/T4aN2M0 & $60.0(34.0-86.0)$ & $47.8(39.8-55.8)$ \\
Remaining in IIIB & T4bN1M0/T3N3aM0 & $39.0(1.2-76.8)$ & $33.7(6.8-60.6)$ \\
Migration from IIIC to IIIB & & & \\
Remaining in IIIB & T4bN1M0/T3N3aM0 & $39.0(1.2-76.8)$ & $33.7(6.8-60.6)$ & 0.014 \\
IIIC to IIIB & T4aN3aM0/T4bN2M0 & $35.0(26.8-43.2)$ & $31.4(23.4-39.4)$ \\
Remaining in IIIC & T4bN3aM0/T4bN3bM0/T4aN3bM0 & $22.0(16.2-27.8)$ & $17.1(8.3-25.9)$ \\
\hline
\end{tabular}

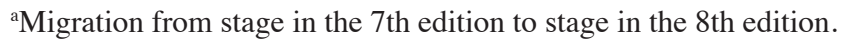

Univariate and multivariate analyses for $O S$ in the two staging systems. Univariate analysis was performed to evaluate the risk factors of OS (Table I). Variables including age, classification of comorbidities, primary tumor size, serum CEA, serum CA 19-9, type of gastrectomy, resection extent, differentiation degree, positive resection margin, pathological $\mathrm{T}$ and $\mathrm{N}$ stage, 7th TNM stage and 8th TNM stage were identified as prognostic factors of OS. After elimination of the variables highly related to others, two Cox proportional regression models were constructed, and included age, classification of comorbidities, primary tumor size, serum CEA, serum CA 19-9, type of gastrectomy, tumor differentiation, positive resection margin and TNM stage in the two staging systems to predict the prognosis of gastric cancer patients (Table IV). In both models, an elevated level of serum CA 19-9, total gastrectomy, positive resection margin and high TNM stage were independent risk factors for predicting an unfavorable OS. A smaller value of -2log likelihood was calculated in the model for the 8th edition of the staging system ( 7 th edition $4,738.859$ vs. 8 th edition $4,736.683$ ), which indicated a better predictive capability compared with the 7 th edition.

\section{Discussion}

The TNM staging system for gastric cancer has been widely used as a method for staging gastric cancer patients and is considered as the most important reference in multimodal treatment. It is also useful for determining the extent of the disease, providing guidance for treatment planning and predicting outcomes. The latest (8th) edition of the AJCC TNM staging system for gastric cancer provides additional resources that are not available in the 7 th edition. The modifications introduced in the 8th edition were based on the clinicopathological and follow-up data from $>25,000$ gastric cancer patients in the International Gastric Cancer Association database, which includes both Asian and Western patients who underwent surgical resection with adequate lymphadenectomy and pathological assessment and were followed up for at least 5 years (6). However, the prognostic value of this new staging system remains unknown. Recently, several analyses based on a similar scale of cohort were also performed. However, their conclusion that the 8th TNM edition may not provide better accuracy in predicting the prognosis of stage III gastric cancer is opposite to the findings of the present study. This also suggested a controversy regarding the superiority of the 8th edition and a large-scale, well-designed study is required to further confirm the conclusions, until which time retrospective analyses from different centers, including the present study, may provide insights into this issue (7-9). In the present study, the suitability of these modifications in the latest 8th edition TNM staging system were evaluated.

Prior to the publication of the 8th edition, there were several issues with the 7th TNM staging system that were raised in numerous studies (10-15). Limitations to $\mathrm{N}$ category classification in the 7 th edition were validated, and the need for relevant modifications, including the application of $\mathrm{N} 3 \mathrm{a} / \mathrm{b}$ to the final staging, had been put forward by several investigators (14,16-19). Through the subdivision of N3 into N3a (MLNC 7-14) and N3b (MLNC $\geq 15$ ), the new staging system emphasizes the importance of the sufficient resection of lymph nodes to avoid understaging and to ensure accurate staging, which had also been previously claimed (20). Ji et al (21) performed a retrospective analysis for a cohort of 1,663 patients with clear eligibility criteria. The authors generally concluded that the 8th edition system is superior to the 7 th edition system in terms of homogeneity, discriminatory ability and monotonicity of gradients for Chinese patients with gastric cancer, based on a sequence of reasonable analyses. However, the sample sizes for $\mathrm{pN} 3 \mathrm{a}$ and $\mathrm{pN} 3 \mathrm{~b}$ were relatively small (30 patients in $\mathrm{pN} 3 \mathrm{a}$ and 6 in pN3b), although significant differences in the 5-year survival rate were found between the two groups. In our current cohort, 244 patients were in the N3a and 122 in the N3b category, from which a more statistically effective comparison could be performed. Our results revealed a lower OS in patients with N3a compared with those with N3b, which was consistent with the findings from previous reports $(17,19,22)$. In the present study, the $\mathrm{N}$ category with the $\mathrm{N} 3$ subdivision also exhibited an improved discriminatory ability on survival curves compared with that without the N3 subdivision. In the 7th edition, although N3 is subdivided into N3a and N3b, they are grouped together in the final TNM staging, which may be not conducive to properly predict stage-based prognosis. Through adopting $\mathrm{N} 3 \mathrm{a} / \mathrm{b}$ in the final TNM staging in the 
Table IV. Multivariable analysis of factors associated with overall survival in the two staging systems.

\begin{tabular}{|c|c|c|c|c|}
\hline \multirow[b]{2}{*}{ Factors } & \multicolumn{2}{|c|}{ 7th edition } & \multicolumn{2}{|c|}{ 8th edition } \\
\hline & Hazard ratio $(95 \% \mathrm{CI})$ & P-value & Hazard ratio $(95 \% \mathrm{CI})$ & P-value \\
\hline Age (years) & & 0.110 & & 0.170 \\
\hline$<60$ & (Reference) & & (Reference) & \\
\hline$\geq 60$ & $1.186(0.962-1.461)$ & & $1.158(0.939-1.427)$ & \\
\hline Classification of comorbidities & & 0.224 & & 0.223 \\
\hline 0 & (Reference) & & (Reference) & \\
\hline 1 & $1.058(0.812-1.377)$ & 0.677 & $1.055(0.810-1.374)$ & 0.691 \\
\hline$\geq 2$ & $1.431(0.951-2.152)$ & 0.085 & $1.433(0.953-2.154)$ & 0.084 \\
\hline Primary tumor size (cm) & & 0.322 & & 0.375 \\
\hline$\leq 5$ & (Reference) & & (Reference) & \\
\hline$>5$ & $1.114(0.900-1.380)$ & & $1.103(0.889-1.368)$ & \\
\hline Serum CEA & & 0.493 & & 0.405 \\
\hline Normal & (Reference) & & (Reference) & \\
\hline Elevated & $1.098(0.840-1.437)$ & & $1.121(0.857-1.465)$ & \\
\hline Serum CA 19-9 & & 0.009 & & 0.012 \\
\hline Normal & (Reference) & & (Reference) & \\
\hline Elevated & $1.362(1.079-1.720)$ & & $1.350(1.069-1.705)$ & \\
\hline Gastrectomy type & & 0.015 & & 0.035 \\
\hline Subtotal & (Reference) & & (Reference) & \\
\hline Total & $1.304(1.052-1.615)$ & & $1.158(0.939-1.427)$ & \\
\hline Differentiation & & 0.359 & & 0.228 \\
\hline High/moderate & (Reference) & & (Reference) & \\
\hline Poor/signet-ring cell carcinoma & $1.141(0.861-1.513)$ & & $1.189(0.897-1.575)$ & \\
\hline Positive resection margin & & 0.002 & & 0.001 \\
\hline No & (Reference) & & (Reference) & \\
\hline Yes & $1.989(1.296-3.052)$ & & $2.001(1.305-3.069)$ & \\
\hline TNM stage & & $<0.001$ & & $<0.001$ \\
\hline IA & (Reference) & & (Reference) & \\
\hline IB & $1.205(0.340-4.274)$ & 0.772 & $1.209(0.341-4.286)$ & 0.769 \\
\hline IIA & $3.336(1.184-9.404)$ & 0.023 & $3.325(1.180-9.374)$ & 0.023 \\
\hline IIB & $3.851(1.636-9.062)$ & 0.002 & $3.829(1.625-9.021)$ & 0.002 \\
\hline IIIA & $5.325(2.258-12.558)$ & $<0.001$ & $6.332(2.769-14.479)$ & $<0.001$ \\
\hline IIIB & $7.347(3.172-17.017)$ & $<0.001$ & $10.452(4.515-24.196)$ & $<0.001$ \\
\hline IIIC & $12.264(5.340-28.163)$ & $<0.001$ & $16.057(6.754-38.176)$ & $<0.001$ \\
\hline IV & $21.591(9.382-49.690)$ & $<0.001$ & $21.801(9.474-50.169)$ & $<0.001$ \\
\hline$-2 \log$ likelihood & 4738.859 & $<0.001$ & 4736.683 & $<0.001$ \\
\hline
\end{tabular}

CI, confidence interval; CEA, carcinoembryonic antigen; CA, cancer antigen.

8th edition, stage migration occurred in four subcategories (T4aN3aM0, T3N3bM0, T2N3bM0 and T1N3bM0), three of which (T3N3bM0, T2N3bM0 and T1N3bM0) migrated to a higher tier, and one (T4aN3aM0) to a lower tier. However, there was only a small number of patients with stage migration to a higher tier ( 2 in T1N3bM0, 4 in T2N3bM0 and 8 in T3N3bM0), suggesting only a small proportion of patients would be moved to a higher tier category by the N3 subdivision in the new staging system. T4aN3aM0, a subgroup of T4, was integrated with the other three $\mathrm{T} 4$ subgroups with stage migration
(T4bN0M0, T4aN2M0 and T4bN3aM0) for analysis. All four of these subgroups were re-classified in stage III, appearing as predominant changes resulting from modifications in the 8th TNM. The changes were divided into two aspects: Re-classification of T4bN0M0 and T4aN2M0 of stage IIIB into IIIA; and re-classification of T4aN3aM0 and T4bN2M0 of stage IIIC into IIIB. The survival comparison demonstrated that patients with T4bN0M0 and T4aN2M0 yielded a more similar median OS and 5-year OS rate to those remaining in stage IIIA (median OS, 60 vs. 68 months; 5-year OS rate, 47.8 
vs. $52.3 \%$, respectively), in comparison with patients remaining in stage IIIB (median OS, 60 vs. 39 months; 5-year OS rate, 47.8 vs. $33.7 \%$, respectively). These findings suggest that it is reasonable to classify T4bN0M0 and T4aN2M0 into stage IIIA rather than stage IIIB. Similar results were also observed in the T4aN3aM0 and T4bN2M0 groups, which were moved from stage IIIC to stage IIIB. By subsequent analysis with survival curves and multivariable Cox proportional regression model, the 8th TNM was demonstrated to be superior to the 7th TNM in predictive capacity by distinctly discriminating survival time and rate in patients with stage IIIA from those in stage IIIB. Kim et al analyzed the stage distribution and migration for the AJCC 7th and 8th editions of the staging system based on a cohort of 5,507 patients from Korea (23). Although the final conclusion that the 8th edition represents a better refinement of the 7 th staging system was similar to ours, their main finding of improved survival discrimination between IIIB and IIIC in the 8th staging system was different from our results, which demonstrated a good discriminatory ability among IIIA through IIIB. Another study by Fang et al conducted a comparative analysis of overall and disease-free survival based on the 7 th and 8 th editions. Although a better homogeneity of the 8th edition was indicated, which was similar to our results, by a calculated higher likelihood ratio Chi-squared statistic (728.51 for the 7th and 740.13 for the 8th edition; $\mathrm{P}<0.001$ ), their study failed to perform further analysis for subgroups of stage III, in which the main stage migration occurred, and contributed to the main modification of the 8th edition (24). Therefore, the 8th edition staging system appears to be more reasonable and accurate for predicting the prognosis of patients with gastric cancer. However, there remain questions regarding the current staging system. For example, it is unclear whether molecular findings (such as HER2 immunoreactivity) should be considered in the staging of gastric cancer, or whether these findings should only be considered as an additional prognostic factor.

There were several limitations to the present study. First, this study represents a retrospective analysis of patients from a single center who received individualized adjuvant chemotherapy without a standard protocol. Second, three groups (T1N3bM0, T2N3bM0 and T3N3bM0) were not statistically analyzed due to the small sample size. These limitations may be overcome by a larger-scale study in future investigations. Third, the follow-up duration was relatively short. Thus, studies with a longer follow-up (for example, 10 years) are required to confirm and extend the findings in this study with certainty. However, despite these limitations, our findings provide an important insight into the application of the new edition of the AJCC TNM staging system for gastric cancer in China.

In conclusion, the subdivision of $\mathrm{N} 3$ into $\mathrm{N} 3 \mathrm{a}$ and $\mathrm{N} 3 \mathrm{~b}$ resulted in a clearer prognostic discrepancy. A sufficient number of resected lymph nodes is important to avoid understaging according to the 8th AJCC TNM edition staging system. The 8th edition of the AJCC TNM staging system appears to be more reasonable, and it is superior to the 7 th edition in predicting OS in gastric cancer patients.

\section{Acknowledgements}

Not applicable.

\section{Funding}

The present study was supported by the State's Key Project of Research and Development Plan (grant nos. 2017YFC0108300 and 2017YFC0108301), the National Natural Science Foundation of China (grant no. 81672446), the Guangdong Provincial Science and Technology Key Project (grant no. 2014A02015014), the Research Fund of Public Welfare in the Health Industry, the National Health and Family Planning Commission of China (grant no. 201402015), the Southern Medical University Clinical Research Start-Up Project (grant no. LC2016ZD003), the Guangzhou Science and Technology Project (grant no. 201400000004-5) and the Key Clinical Specialty Discipline Construction Program [grant no. (2011)170].

\section{Availability of data and materials}

The datasets generated and analyzed in the present study are available for the corresponding author upon reasonable request.

\section{Authors' contributions}

HW and WG made substantial contributions to data collection and were major contributors in analyzing data and writing the manuscript. TM and LZ were responsible for the maintaining the clinical database and acquisition and interpretation of data. TL, JY, YH, HC and TL made substantial contributions to data collection, data interpretation, and made critical revision of the manuscript. HL and GL made substantial contributions to the design and general supervision of the present study. All the authors have read and approved the final version of the manuscript.

\section{Ethics approval and consent to participate}

The study was approved by the Ethics Committee of Nanfang Hospital of Southern Medical University. Written informed consent was obtained from all the patients prior to entering their information into the database.

\section{Patient consent for publication}

Not applicable.

\section{Patient competing interests}

The authors declare that they have no competing interests to disclose.

\section{References}

1. Torre LA, Bray F, Siegel RL, Ferlay J, Lortet-Tieulent J and Jemal A: Global cancer statistics, 2012. CA Cancer J Clin 65: 87-108, 2015

2. Wittekind C: The development of the TNM classification of gastric cancer. Pathol Int 65: 399-403, 2015.

3. Sobin LH, Gospodarowicz MK and Wittekind C (eds); International Union Against Cancer (UICC): TNM Classification of Malignant Tumors. 2nd edition. Springer, Gneva, 1974.

4. Washington K: 7th edition of the AJCC cancer staging manual: stomach. Ann Surg Oncol 17: 3077-3079, 2010. 
5. Hu YF, Yu J, Zhang C, Wang YN, Cheng X, Huang F and Li GX Development and implementation of a clinical data mining system for gastric cancer surgery. Zhonghua wei chang wai ke za zhi 13:510-515, 2010 (In Chinese).

6. Sano T, Coit DG, Kim HH, Roviello F, Kassab P, Wittekind C, Yamamoto, Y and Ohashi, Y: Proposal of a new stage grouping of gastric cancer for TNM classification: International Gastric Cancer Association staging project. Gastric cancer 20:217-225, 2017.

7. Lu J, Zheng CH, Cao LL, Ling SW, Li P, Xie JW, Wang JB, Lin JX, Chen QY, Lin M, Tu RH and Huang CM: Validation of the American Joint Commission on Cancer (8th edition) changes for patients with stage III gastric cancer: survival analysis of a large series from a Specialized Eastern Center. Cancer Med 6 : 2179-2187, 2017.

8. Lu J, Zheng CH, Cao LL, Li P, Xie JW, Wang JB, Lin JX, Chen QY, Lin M, Tu RH and Huang CM: Comparison of the 7th and 8th editions of the American joint committee on cancer TNM classification for patients with stage III gastric cancer. Oncotarget 8: 83555-83562, 2017.

9. Lu J, Zheng CH, Cao LL, Li P, Xie JW, Wang JB, Lin JX, Chen QY, Lin M and Huang CM: The effectiveness of the 8th American Joint Committee on Cancer TNM classification in the prognosis evaluation of gastric cancer patients: A comparative study between the 7th and 8th editions. Eur J Surg Oncol 43 2349-2356, 2017

10. Fang WL, Huang KH, Chen JH, Lo SS, Hsieh MC, Shen KH, $\mathrm{Li} \mathrm{AF}$, Niu DM, Chiou SH, et al: Comparison of the survival difference between AJCC 6th and 7th editions for gastric cancer patients. World J Surg 35:2723-2729, 2011.

11. Wang W, Sun XW, Li CF, Lv L, Li YF, Chen YB, Xu DZ, Kesari R, Huang CY, et al: Comparison of the 6th and 7th editions of the UICC TNM staging system for gastric cancer: results of a Chinese single-institution study of 1,503 patients. Ann Surg Oncol 18: 1060-1067, 2011.

12. Qiu MZ, Wang ZQ, Zhang DS, Liu Q, Luo HY, Zhou ZW, Li YH, Jiang WQ and Xu RH: Comparison of 6th and 7th AJCC TNM staging classification for carcinoma of the stomach in China. Ann Surg Oncol 18: 1869-1876, 2011.

13. Kikuchi S, Futawatari N, Sakuramoto S, Katada N, Yamashita K, Shibata T, Nemoto $\mathrm{M}$ and Watanabe M: Comparison of staging between the old (6th edition) and new (7th edition) TNM classifications in advanced gastric cancer. Anticancer Res 31: 2361-2365, 2011

14. Marano L, Boccardi V, Braccio B, Esposito G, Grassia M, Petrillo M, Pezzella M, Porfidia R, Reda G, et al: Comparison of the 6th and 7th editions of the AJCC/UICC TNM staging system for gastric cancer focusing on the " $\mathrm{N}$ " parameter-relate survival: the monoinstitutional NodUs Italian study. World J Surg Oncol 13: 215, 2015

15. Ilhan E, Ureyen O and Meral UM: Ongoing problems concerning 7th TNM Staging System and Proposals for 8th TNM Staging System of Gastric Cancer. Prz Gastroenterol 11:223-225, 2016.
16. Saito H, Fukumoto Y, Osaki T, Fukuda K, Tatebe S, Tsujitani S and Ikeguchi M: Prognostic significance of level and number of lymph node metastases in patients with gastric cancer. Ann Surg Oncol 14:1688-1693, 2007.

17. Marrelli D, Morgagni P, de Manzoni G, Coniglio A, Marchet A, Saragoni L, Tiberio G and Roviello F: Prognostic value of the 7th AJCC/UICC TNM classification of noncardia gastric cancer: analysis of a large series from specialized Western centers. Ann Surg 255: 486-491, 2012.

18. Jung H, Lee HH, Song KY, Jeon HM and Park CH: Validation of the seventh edition of the American Joint Committee on Cancer TNM staging system for gastric cancer. Cancer 117: 2371-278, 2011.

19. Ahn HS, Lee HJ, Hahn S, Kim WH, Lee KU, Sano T, Edge SB and Yang HK: Evaluation of the seventh American Joint Committee on Cancer/International Union Against Cancer Classification of gastric adenocarcinoma in comparison with the sixth classification. Cancer 116: 5592-5598, 2010.

20. Karpeh MS, Leon L, Klimstra D and Brennan MF: Lymph node staging in gastric cancer: is location more important than Number? An analysis of 1,038 patients. Ann Surg 232: 362-371, 2000.

21. Ji X, Bu ZD, Yan Y, Li ZY, Wu AW, Zhang LH, Zhang J, Wu XJ, Zong XL, Li SX, Shan F, Jia ZY and Ji JF: The 8th edition of the American Joint Committee on Cancer tumor-node-metastasis staging system for gastric cancer is superior to the 7th edition: results from a Chinese mono-institutional study of 1663 patients. Gastric Cancer 21: 643-652, 2018.

22. Sun Z, Wang ZN, Zhu Z, Xu YY, Xu Y, Huang BJ, Zhu GL and $\mathrm{Xu}$ HM: Evaluation of the seventh edition of American Joint Committee on Cancer TNM staging system for gastric cancer: results from a Chinese monoinstitutional study. Ann Surg Oncol 19: 1918-1927, 2012.

23. Kim SG, Seo HS, Lee HH, Song KY and Park CH: Comparison of the Differences in Survival Rates between the 7 th and 8th Editions of the AJCC TNM Staging System for Gastric Adenocarcinoma: a Single-Institution Study of 5,507 Patients in Korea. J Gastric Cancer 17: 212-219, 2017.

24. Fang WL, Huang KH, Chen MH, Liu CA, Hung YP, Chao Y, Tai LC, Lo SS, Li AF, Wu CW and Shyr YM: Comparative study of the 7th and 8th AJCC editions for gastric cancer patients after curative surgery. PLoS One 12: e0187626, 2017. 